\title{
La création contemporaine comme muséologie participative, le musée comme espace de spectacle vivant
}

Contemporary creativity as participatory museology: the museum as a space for living exhibitions

\section{Nathalie Noël-Cadet et Céline Bonniol}

\section{OpenEdition}

Journals

\section{Édition électronique}

URL : http://journals.openedition.org/iss/467

DOI : $10.4000 /$ iss. 467

ISSN : 2306-4161

\section{Éditeur}

ICOM - International Council of Museums

\section{Édition imprimée}

Date de publication : 1 décembre 2015

Pagination : 185-194

ISSN : 2309-1290

Référence électronique

Nathalie Noël-Cadet et Céline Bonniol, « La création contemporaine comme muséologie participative, le musée comme espace de spectacle vivant », ICOFOM Study Series [En ligne], 43b | 2015, mis en ligne le 06 février 2018, consulté le 23 avril 2019. URL : http://journals.openedition.org/iss/467 ; DOI : $10.4000 /$ iss. 467 


\title{
La création contemporaine comme muséologie participative, le musée comme espace de spectacle vivant
}

\author{
Nathalie Noël-Cadet \\ Maître de conférences en sciences de l'information et de la \\ communication au laboratoire LCF-EA 4549. Université de La \\ Réunion - France \\ Céline Bonniol \\ Docteur en sciences de l'information et de la communication, \\ chercheur associé au laboratoire LCF-EA 4549. Université de \\ La Réunion - France
}

\section{Introduction}

La construction de dispositifs liés au spectacle vivant et de projets de création contemporaine pour proposer un éclairage différent sur une collection ou sur un lieu de patrimoine est une situation de plus en plus fréquente. A titre d'exemple, après Tony Cragg et Wim Delvoye, c'est Loris Gréaud qui investit cette année le musée du Louvre. Autre haut lieu du patrimoine français, le château de Versailles invite depuis plusieurs années un artiste contemporain à exposer dans ses murs et ses jardins. La création contemporaine, qu'elle relève des arts plastiques et visuels ou du spectacle vivant devient, dans certains cas un objet hybride, au carrefour entre médiation et création.

Notre communication souhaite présenter ce processus dans le contexte développement culturel que connaît La Réunion depuis quelques années (Magdelaine-Andrianjafitrimo, 2011). Malgré une situation de crise qui handicape les compagnies et les artistes et voit les budgets de subventions diminuer, l'offre artistique et culturelle sur le territoire réunionnais a significativement augmenté ces dernières années. Des équipements culturels ont vu le jour, d'autres sont en devenir, les programmations s'étoffent. La Réunion est pourvue de différents outils pour le développement de l'art contemporain sur son territoire : une école d'art proposant le cursus complet et formant chaque année une nouvelle vague de jeunes plasticiens, un FRAC, une intéressante collection d'art moderne et contemporain au musée Léon Dierx, une Artothèque, un petit réseau actif de structures associatives. Si l'île souffre d'un manque de lieux de diffusion pour la création plastique et visuelle, les acteurs du paysage culturel réunionnais inventent des alternatives afin de rendre possible la rencontre entre la création et les publics : investissement de l'espace public, création d'événements, ouverture des espaces culturels non dédiés à la création contemporaine aux propositions des artistes actuels. Des projets ambitieux sont en cours comme la création d'un Centre d'art dans le Sud de l'île, et d'une Cité des arts dotée d'ateliers d'artistes dans le Nord.

Dans ce contexte de développement, de quelle manière les dispositifs de médiation sont-ils conçus pour rendre la muséologie vivante ? Quel est le rôle de l'action culturelle et de la création contemporaine dans la construction de nouvelles expériences muséales ? Quels sont les modes de connexion entre différents champs: musée d'histoire et art contemporain, espace muséal et 
spectacle vivant? Quels nouveaux discours sont générés par ces connexions, nous permettant d'aborder le concept de « muséologie participative » sous l'angle de l'art contemporain ?

Nous répondrons à ces questions à travers l'analyse de plusieurs exemples réunionnais: les propositions artistiques de deux jeunes plasticiens à l'occasion de la manifestation Nuit d'art de Pleine Lune proposant une relecture des collections du musée historique de Villèle et celle d'une autre plasticienne qui interroge dans son travail les rapports entre le champ muséal, le champ du spectacle vivant et celui de l'art contemporain.

\section{"La sollicitation par participation »: comment l'art contemporain vient-il questionner «la muséologie participative »?}

Le terme de "muséologie participative " se manifeste officiellement dans les musées dans les années 1970 autour du « désir d'associer les visiteurs potentiels à la programmation des musées pour qu'ils soient bien adaptés à leur public " (Merleau-Ponty, 2006, p. 239). Son histoire liée au mouvement des écomusées et de la nouvelle muséologie questionne le nouveau positionnement des musées vis-àvis de l'intégration des visiteurs dans leur mise en scène muséographique pour des enjeux politiques de démocratisation culturelle (Le Marec, 2006, p. 259). De ce fait, le questionnement sur " la muséologie participative » s'est essentiellement orienté autour de ces aspects au détriment de l'émergence des objets et des formes artistiques qui se sont développées autour de «la participation par sollicitation" (Chaumier, 2006, p.249). Ce concept est utilisé par Serge Chaumier pour expliquer qu'il existe en parallèle aux écomusées d'autres initiatives menées par des collectifs d'artistes questionnant la « muséologie participative » par la place accordée au public dans l'acte de création même.

Pourtant, l'art contemporain dès les années 60 a bouleversé l'univers traditionnel des musées en y intégrant de nouvelles formes artistiques hybrides plaçant le public au cœur de la création artistique. Comme l'indique Marion Viollet, les artistes du XX $\mathrm{X}^{\mathrm{e}}$ siècle conscients de la passivité du public face à une œuvre d'art tentent de :

faire appel à la participation physique du visiteur, invité à toucher les pièces, les plasticiens le font intervenir dans la création même de l'œuvre, voire dans le développement de sa démarche (Viollet, 2011, p. 15).

Les spectateurs ne sont plus passifs mais deviennent "acteurs du déroulement de la création " et sont invités à "devenir sujet et ou/acteur de leur production ". L'art contemporain questionne à la fois le rapport au public et à la mise en exposition.

Ces premiers signes de l'évolution de l'attitude du public apparaissent visiblement lorsqu'il devient spectateur de performances avec les mouvements futuristes, constructivistes, dadaïstes et surréalistes: « la performance est une façon d'en appeler directement au public, de heurter l'auditoire pour l'amener à réévaluer sa propre conception de l'art et de ses rapports avec la culture " (Goldberg, 2001, p. 8). Pour ces mouvements tant au théâtre qu'en peinture, le spectateur 
avait un nouveau rôle. II était mis au travail parfois dans ces formes expressives les plus violentes

$\mathrm{DaDa}$ interpelle le public, provoque sa réaction dans les performances. Les dadaïstes parviennent à modifier l'attitude passive des regardeurs en autorisant les cris, les injonctions, les lancers de projectiles au cours de leurs pièces de théâtre. Ce premier pas met à mal la distance séparant l'artiste et la création, de celui qui observe et admire. II sera accompagné de plusieurs gestes de désacralisation de l'œuvre d'art (Viollet, 2011, p. 76).

De ce fait, le musée devient aussi par l'intermédiaire de l'art contemporain un lieu où nous observons les logiques de l'espace public comme sphère d'apparition et espace énonciatif. La crise de l'art contemporain dans les années 1990 ainsi que les nombreux scandales lui étant associés avaient mis de côté l'acte de création participative des performances, laissant place à «la figure du médiateur » (Bonniol, 2009). Dans les années 2000 s'opère un retour dans la sphère muséale de la performance comme espace d'énonciation

\begin{abstract}
Tout un courant de la performance actuelle semble renouer avec une certaine théâtralité, caractérisée par une prééminence de la parole. Si les formes orales ont accompagné l'histoire de la dématérialisation de l'art, de plus en plus d'artistes font de l'énonciation un enjeu majeur de leur travail à travers des lectures, visites guidées, conférences et autres "savoureuses surprises "... Les artistes prennent la parole: comme un acte politique d'abord se réappropriant les formes du discours qui relèvent habituellement de la compétence d'experts en tous genres. (Pellegrin, 2010, p. 73).
\end{abstract}

C'est le musée comme espace de nouvelles médiations énonciatives, par l'art contemporain, que nous avons voulu analyser sur le terrain réunionnais en posant comme hypothèse que celui-ci permet d'aborder la muséologie participative par une approche plus culturelle que sociale.

\title{
L'art contemporain comme nouvelle forme énonciative d'une muséologie participative
}

Le contexte de La Réunion pour cette analyse nous a semblé intéressant, par l'histoire récente de la création contemporaine. L'absence, sur l'île, d'un nombre important de lieux dédiés à la création contemporaine a influencé la mise en place de dispositifs innovants comme les événementiels et l'intégration de l'art contemporain dans des espaces muséaux ne lui étant pas dédiés à l'origine du projet muséologique.

Pour notre étude, nous avons sélectionné deux terrains différents nous permettant d'approfondir les modalités énonciatives issues des objets et formes artistiques qui se développent autour de ce que Serge Chaumier (2006, p.249) appelle «la participation par sollicitation » pour interroger sous un autre angle « la muséologie participative $»$. 
Le premier terrain sélectionné est une manifestation d'art contemporain La Nuit d'art de Pleine Lune ${ }^{81}$ relevant d'un événementiel.

Le second est une œuvre d'art contemporain intégrant les codes du spectacle vivant au musée.

Le premier terrain de l'étude, La Nuit d'art de Pleine Lune: l'évenementiel d'art contemporain

La Nuit d'art de Pleine Lune ${ }^{82}$ est un concept d'évènement créé par l'association Cheminement(s), structure associative de l'lle de La Réunion $^{83}$. II propose un mariage entre le patrimoine et la création plastique contemporaine en invitant des artistes plasticiens et visuels à investir un site patrimonial de façon éphémère, une nuit par an, avec une production artistique originale et in situ. Depuis 2011, La Nuit d'art de Pleine Lune a lieu au musée historique de Villèle ; c'est un site historique et symbolique pour l'histoire de La Réunion.

Ce lieu, marqué par les époques de l'esclavage, de l'engagisme, de l'industrie sucrière, est aujourd'hui un musée, le musée d'histoire de La Réunion. Plusieurs corps de bâtiments peuvent se visiter, ainsi que les jardins. Certains espaces sont consacrés à des expositions temporaires; d'autres espaces proposent une exposition permanente : les cellules de l'ancien hôpital des esclaves accueillent un mémorial et une exposition documentaire sur panneau présentant la vie des esclaves. Le rez-de-chaussée de ce qui était à l'origine l'habitation principale du domaine, construite à l'époque de la famille Desbassyns, grands propriétaires terriens des $\mathrm{XVIII}{ }^{\mathrm{e}}$ et $\mathrm{XIX}{ }^{\mathrm{e}}$ siècles, propose un parcours de visite suivant les espaces fonctionnels domestiques : entrée, petit salon, cabinet de travail, chambre, grand salon, salle à manger.

Pour La Nuit d'art de Pleine Lune, le site du musée de Villèle sert de cadre et de source d'inspiration à la création. En retour la création, en s'intégrant sur le site propose, une nouvelle approche d'une histoire habituellement rapportée à travers des objets et des visites guidées. L'art contemporain, en intégrant des espaces muséaux, amène une lecture différente de la muséologie traditionnelle à travers des médiations énonciatives impliquant des formes de participation à différents niveaux des publics. Les trois exemples choisis ici nous permettent de saisir trois formes participatives que propose l'art contemporain pour une lecture différente de l'espace muséal.

Parmi les différentes œuvres présentes lors de La Nuit d'art de Pleine Lune, nous avons sélectionné deux œuvres proposant des formes énonciatives différentes pour une nouvelle lecture de l'histoire du musée.

Exemple 1: Ombline d'Alice Aucuit, Nuit d'art de Pleine Lune 2013 Ombline est le titre d'une installation composée de pièces en céramique, réalisée à l'occasion de La Nuit d'art de Pleine Lune 2013. Ombline est également le prénom de Mme Desbassyns, figure emblématique de La Réunion du début du $19{ }^{\text {ème }}$ siècle, grande propriétaire terrienne et habitante de la maison qui deviendra en 1974 le musée de Villèle.

L'installation Ombline se présente comme un récit mystère. La plasticienne Alice Aucuit a souhaité traiter du personnage de Mme

\footnotetext{
${ }^{81} \mathrm{http} / / /$ www.cheminements.org/napl-def.html

$82 \mathrm{http}: / /$ www.cheminements.org/napl-def.html

83 http://www.cheminements.org/
} 
Desbassyns. A travers différents éléments, elle reconstitue un moment de vie du personnage dans sa maison. L'artiste a choisi d'investir la pièce qui correspondait à la chambre de Mme Desbassyns. Le parcours muséographique du Musée de Villèle suit les différentes pièces de l'ancienne habitation, composées du mobilier d'origine et d'autres objets venus s'ajouter au parcours au fil du temps et des propriétaires successifs.

L'intervention artistique dans le cadre de La Nuit d'art de Pleine Lune amène une dimension narrative supplémentaire à l'espace muséal. Le visiteur, d'ordinaire guidé par le discours du médiateur présentant les différents objets se trouvant dans le lieu, peut, à l'occasion de cet événement, laissé aller son imagination en prenant appui sur une œuvre contemporaine. L'artiste a disposé dans plusieurs endroits de la chambre des flacons de céramique peints. Elle les a positionnés de façon à donner l'impression qu'ils ont été renversés sur le dessus de la commode, sur le lit ou à même le sol. Une grosse araignée en céramique est placée sur le lit de Mme Desbassyns. La plasticienne fait ici référence à une grande artiste qu'elle admire, Louise Bourgeois. Cette dernière a réalisé en 1999 une sculpture monumentale représentant une araignée et faisant référence à la figure de la mère. En produisant une araignée renvoyant au personnage de Mme Desbassyns, Alice Aucuit fait écho au personnage en tant que mère.

Avec son installation, la plasticienne propose un autre visage de Mme Desbassyns ; elle l'aborde et la donne à imaginer non plus seulement comme une propriétaire ayant tenu seule et d'une main de fer, pendant quarante ans après la mort de son mari, sa propriété et ses très nombreux esclaves, mais aussi comme une femme dans un quotidien féminin et maternel. L'œuvre amène ici un autre point de vue qui n'est d'ordinaire pas transmis par le discours muséographique.

Exemple 2: Murmure de Félix Duclassan, Nuit d'art de Pleine Lune 2013.

Le parcours de visite du musée de Villèle se termine par l'office, pièce qui servait à la préparation des plats servis ensuite dans la salle à manger la jouxtant, lorsque la maison était habitée. C'est dans cet espace que le plasticien Félix Duclassan, toujours à l'occasion de La Nuit d'art de Pleine Lune 2013, a souhaité présenter son travail.

Le jeune artiste s'est intéressé non pas aux personnages qui sont généralement cités lors des visites guidées du Musée de Villèle, mais aux personnages anonymes qui ont, eux aussi, vécus dans ces lieux: couturière, cuisinier, hommes et femmes de maison. Son travail s'est effectué en plusieurs temps : d'abord, l'artiste est allé à la rencontre des descendants de ces personnages dont les familles, pour beaucoup, habitent toujours aujourd'hui dans le quartier de Villèle tout proche du musée. II a procédé à une collecte de mémoire en écoutant le récit des personnes rencontrées et en récupérant différents documents et images, dont des portraits des dernières personnes ayant travaillé dans la maison Desbassyns au $X X^{e}$ siècle, avant que le Conseil Général de La Réunion ne l'acquière pour en faire un musée. A partir de ces images, l'artiste, ayant une pratique du dessin, réalisa un portait de deux personnages. Ce dessin en grand format a servi de modèle pour un travail de broderie, étape de la production pour laquelle l'artiste a demandé la collaboration d'un groupe de brodeuses, habitant le quartier de Villèle et pour certaines d'entre elles ayant côtoyé les personnages portraiturés sur l'ouvrage. 
II s'agit donc d'un travail à plusieurs mains pour lequel l'artiste intervient comme un opérateur, l'initiateur d'un programme de création faisant intervenir plusieurs individus cumulant les rôles de témoins, acteurs, spectateurs.

La pièce finale présente une œuvre très graphique, épurée. Sur un fond neutre, les deux personnages, parfaitement reconnaissables, se détachent. Aucun décor ou indication de lieux n'est figuré sur l'image ; l'esthétique joue sur l'absence ; les personnages semblent émerger de la matière, comme des apparitions.

La dernière étape de ce processus de création consiste en l'installation du tableau brodé à l'intérieur du musée de Villèle, dans l'office. Cette installation s'accompagne de l'inversement du parcours de visite à l'occasion de La Nuit d'art de Pleine Lune. Habituellement, le visiteur du musée de Villèle commence sa visite guidée par l'entrée qui présente l'arbre généalogique de la famille Desbassyns à laquelle s'intéressera le reste du déroulement de la visite. A travers l'œuvre Murmure de Félix Duclassan, cette habitude est rompue, un autre point de vue sur les lieux visités est montré.

Aujourd'hui, l'œuvre Murmure est devenue un nouvel élément du discours muséographique. La création contemporaine éclaire et construit le discours sur l'histoire ; elle agit comme un outil de mise en patrimoine. Cet exemple modifie le statut de spectateur et présente la création contemporaine comme une médiation muséale.

Le second terrain de l'étude : Fleur bleue, être sentimentale, la visite guidée de Myriam Omar Awadi

Myriam Omar Awadi est une jeune plasticienne vivant et travaillant à La Réunion. Diplômée de l'Ecole supérieure d'art de La Réunion, elle a participé ces dernières années à différentes expositions et résidences de création dans divers endroits du globe.

Son travail actuel s'intègre dans un corpus de travaux intitulé Esthétique de la broderie. La thématique de la broderie est à envisager dans ses multiples significations. Concrètement la broderie renvoie à un travail textile, art de décoration minutieux associé à une pratique féminine, permettant la production d'une image. Dans un sens figuré, la broderie fait référence à une certaine forme de discours, un discours augmenté de détails inventés par l'énonciateur afin de l'embellir. Cette thématique polysémique traduit l'univers ambivalent de l'artiste, une innocence créatrice emplie de finesse et de douceur sous laquelle se cache un méta-discours proposant une réflexion résolument contemporaine sur le milieu artistique et ses codes. Myriam Omar Awadi s'interroge sur la place de l'artiste dans la société, la place du discours dans le monde de l'art.

Filant la métaphore de "l'esthétique de la broderie », son dernier travail est à la croisée de plusieurs champs. Fleur bleue, être sentimentale: la visite guidée est une œuvre conçue comme une performance puis jouée à différentes occasions en étant achetée comme un spectacle. La performance est la visite guidée d'une exposition vide. Le public est invité à entrer dans une salle d'exposition dans laquelle rien n'est visible sur les murs. Un médiateur, joué par un comédien, s'adresse aux visiteurs en contextualisant et en décrivant des tableaux absents. Le publicvisiteur s'appuie sur ce qui est dit pour voir, imaginer ces dessins.

Pour la plasticienne, il s'agit avant tout d'un travail d'écriture. Le rapport au texte, à l'écrit est une dimension de la création qui a 
toujours intéressé l'artiste : "j'ai toujours écrit, mais je ne savais pas comment l'exploiter » dit-elle ${ }^{84}$. Ce sont la mise en scène et la mise en espace d'une parole que recherche l'artiste, à la fois auteure, metteure en scène et scénographe de cette performance. Le texte est mis en jeu, d'où l'importance de faire intervenir un comédien, expert de la forme scénique, fournissant des clés à la mise en image dans l'esprit des visiteurs. Dans un second temps et dans un autre lieu, les visiteurs peuvent voir les dessins, car ceux-ci existent bel et bien. Myriam Omar Awadi développe depuis plusieurs années la pratique du dessin abordée comme une écriture chorégraphique, des dessins narratifs qui s'ouvrent sur des histoires possibles. Dans un espace sans repères, des corps fluides s'entremêlent, l'interprétation est ouverte.

Ainsi, la pièce présentée est une performance, un travail de mise en scène dans lequel l'artiste joue à la fois sur le dispositif théâtral et sur celui de la visite guidée, un espace, un acteur, un texte, orchestrée par une plasticienne qui collabore avec un comédien et, malgré lui, avec un public. En soi la performance artistique propose déjà une hybridité formelle. Développée dans les années 60 , bien que ces

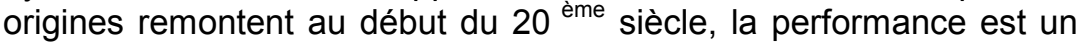
médium interdisciplinaire. Corps, espace, mise en scène, temporalité en sont les ingrédients. La performance interroge les catégories artistiques dans le sens où elle n'est pas une production d'objet, mais la construction d'un moment, d'une situation. C'est pourquoi les artistes contemporains ont souvent recours aux traces vidéographiques ou photographiques pour rendre pérennes leurs performances. Un glissement s'opère entre un temps qui passe et un objet qui reste (Bégoc \& Boulouch \& Zabunyan, 2011, p.15). Dans le cas de la performance Fleur bleue, être sentimentale, l'artiste n'a pas souhaité produire des images permettant de figer la performance dans le temps, et pour cause, cette performance reposant en partie sur la capacité du public à construire lui-même ces images et ce moment. En revanche, un livre a été réalisé avec le texte dit par le comédien au moment de la visite guidée. La performance est donc prolongée par un travail d'édition, par la production d'un livre d'artiste ayant valeur d'objet d'art tout en étant la trace de la performance.

C'est donc sur le texte que s'ouvre cette performance, et qu'elle se referme. Le texte est polymorphe, écrit, joué, édité ; il est le cœur de ce travail. Au-delà de la recherche formelle, le discours permet à l'artiste de véhiculer certaines idées, certains points de vue sur le monde de l'art aujourd'hui en faisant la critique de la place prépondérante que prennent les discours sur l'art et les artistes. Le texte de la performance est également une réflexion sur le discours de médiation. L'artiste s'interroge sur la manière de parler d'une œuvre, sur le parti pris que l'on doit retenir dans le ton, dans la construction; l'œuvre se nourrit de l'étude du processus de médiation.

Le travail de Myriam Omar Awadi vient éclairer notre propos à plus d'un titre. On y trouve un croisement des genres. La pièce qu'elle propose est au carrefour de la performance de la visite guidée du jeu d'acteur. De cette façon, elle souligne les liens présents entre ces différents dispositifs. A quel moment un dispositif scénique devient-il un dispositif de médiation ? A quel moment la production artistique devient-elle outil de médiation? En interrogeant les codes de la production artistique, l'artiste interroge aussi les codes de sa

\footnotetext{
${ }^{84}$ Extrait d'un entretien avec l'artiste Myriam Omar Awadi, avril 2014.
} 
réception qui peut, pour une même proposition, être plurielle. Ce travail montre la porosité existant entre plusieurs formes artistiques et entre les différentes postures du spectateur qui est simultanément spectateur d'une performance et d'une scène jouée, visiteur d'une exposition, participant à la visite. II fait entièrement partie du processus de création; sans sa présence, l'œuvre n'est pas activée, elle n'existe pas ${ }^{85}$.

L'analyse menée à la fois autour de l'événementiel La Nuit d'art de Pleine Lune et de la création La visite guidée de Myriam Omar Awadi nous invite à interroger les formes de sollicitation du public que propose l'art contemporain. Le visiteur n'est plus uniquement "regardeur » ou " participant », il devient aussi " spectateur » dans la mesure où l'artiste intègre les logiques du spectacle vivant jusqu'à sa mise en scène. Venir à La Nuit d'art de Pleine Lune devient une programmation annuelle, où l'on vient "lire », "regarder » autrement le musée, et La visite guidée de Myriam Omar Awadi nous propose d'intégrer par l'intermédiaire de l'écriture et du jeu théâtral le spectacle vivant.

\title{
Conclusion
}

L'art contemporain joue avec les codes, y compris ceux de la muséologie. En construisant de nouveaux modes de production, les œuvres contemporaines deviennent des processus médiatiques; elles proposent de nouvelles expériences muséales. Comme le montrent les exemples sélectionnés pour l'étude, l'art contemporain en intégrant des espaces muséaux apporte une nouvelle approche de l'histoire habituelle rapportée par des visites guidées. Ces nouvelles formes énonciatives, allant de la performance aux installations, amènent une lecture différente de la muséologie traditionnelle où le visiteur devient acteur de la construction du sens.

Ainsi, l'œuvre d'art contemporain offre aussi un espace de liberté d'expression pour les espaces muséaux intégrant le concept de "muséologie participative " ancré dans les logiques du spectacle vivant. Ce sont la mise en scène et la mise en espace d'une parole qui sont au centre de cette "muséologie participative ". Ici, la forme de revendication parfois politique accorde une part plus importante à la liberté laissée au public de construire lui-même son propre discours. Comme l'indique Joëlle Le Marec, la muséologie participative est un enjeu pour les musées pour revendiquer leur rôle social dans l'intégration du visiteur dans la logique de l'institution, mais cette dernière s'est bien souvent développée :

\begin{abstract}
dans un contexte idéologique d'une «explosion de la communication » dans une société d'après-guerre " conquise par la communication ", avec la montée en puissance des médias et la multiplication des métiers de le communication (...) La publicité faite aux innovations à la fois organisationnelles et techniques en matière de démocratie participative est à la mesure de la discrétion qui entoure le contenu des débats ainsi menés. (Le Marec, 2006, p. 266).
\end{abstract}

Dans cette perspective, la réflexion menée sur «la muséologie participative " ne pouvait que s'orienter autour de la logique de la médiation technique où les dispositifs médiatiques participent à la

\footnotetext{
${ }^{85}$ Nous faisons ici référence au concept " d'activation » ou " d'implémentation » de Nelson Goodman.
} 
mise en débat dans les espaces muséographiques. L'exposition pensée comme dispositif médiatique (Davallon, 1999) accompagnant "la muséologie symbolique " et, intégrant une " muséologie de point de vue " a amené à reléguer au second plan l'acte de création de l'artiste comme dispositif pouvant lui aussi proposer une logique participative.

Ainsi interroger "la muséologie participative » en accordant une place à la réflexion sur l'art contemporain nous a permis de sortir de la vision purement fonctionnelle proposée par une «médiation technique ». Notre approche accorde un rôle à «la médiation artistique " dans l'élaboration d'une "muséologie participative » comme espace d'énonciation de la relation artiste/public. Par «cette médiation artistique », l'espace muséal se construit selon la logique " des énoncés performatifs » au sens de Sperber \& Wilson, 1999. Cet espace "ostensivo-inférentiel», au sens de Jean Davallon (1999, p.116), donne à voir et invite le visiteur à se positionner par sa participation.

\section{Références}

Bonniol, C. (2009). L'évolution de la réception des œuvres au cours de visites répétées d'un musée d'art contemporain. Thèse de doctorat en sciences de l'information et de la communication, (sdr) Gottesdiener H. Avignon : Université d'Avignon et des Pays de Vaucluse.

Chaumier, S. (2006). "Le public, acteur de la production d'exposition ? un modèle écartelé entre enthousiasme et réticences. " Dans J. Eidelman, M. Roustan, B. Goldstein (Ed.). La place des publics. De l'usage des études et recherches par les musées. Paris: La Documentation Française.

Davallon, J. (1999). L'exposition à l'œuvre. Stratégie de communication et médiation symbolique. Paris : L'Harmattan.

Goldberg, R. (2001). La performance du futurisme à nos jours. Paris: Thomas \& Hudson.

Le Marec, J. (2006). « Muséologie participative, évaluation, prise en compte des publics : la parole introuvable ». Dans J. Eidelman, M. Roustan, B. Goldstein (Ed.). La place des publics. De l'usage des études et recherches par les musées. Paris : La Documentation française.

Magdelaine-Andrianjafitrimo V. (2011). «Les expressions culturelles et artistiques à la Réunion. "Dans M. Watin \& E. Wolf (Eds.), La Réunion une société en mutation, Univers créole 7, Paris: Economica.

Merleau-Ponty, C. (2006). Les muséologies participatives, associer les visiteurs à la conception des expositions. Dans J. Eidelman, M. Roustan, B. Goldstein (Eds.). La place des publics. De l'usage des études et recherches par les musées. Paris: La Documentation française.

Pellegrin, J. (2010, août, septembre, octobre). « La performance comme espace d'énonciation. " Art Press, 18.

Sperber, D., \& Wilson, D. (1989). La Pertinence : communication et cognition. Paris : Minuit.

Viollet, M. (2011). Les comportements des spectateurs comme enjeux de l'art contemporain. Thèse de doctorat en art plastique, (sdr) Clévenot $D$. \& Buinet, C. Toulouse : Université Toulouse 2.

\section{Résumé}

La problématique de "muséologie participative " s'est longtemps définie depuis les années '70 à travers les enjeux sociopolitiques et identitaires de reconnaissance de "communautés de publics". S'exprimant dans une mise en exposition laissant une large place aux logiques communicationnelles des dispositifs médiatiques, au 
détriment de "la médiation artistique », le concept s'est peu défini autour de "l'esthétique relationnelle » développée dans les années 60 par l'art contemporain. Par cette communication, nous proposons une nouvelle approche de " la muséologie participative » en portant un regard sur les créations contemporaines dont la mise en exposition est pensée selon des logiques proches du spectacle vivant pour jouer sur la relation « visiteur-spectateur-producteur ».

Mots clé : muséologie participative, communication, spectacle vivant

\section{Abstract}

Contemporary creativity as participatory museology: the museum as a space for living exhibitions

The issue of "participatory museology" has been defined since the early 1970s in relation to the socio-political and identity challenges of recognizing "community audiences. Expressing itself through exhibitions that allowed a broad range of communication approaches in media settings, at the expense of "artistic mediation", this concept was scarcely influenced by the "relational esthetic" developed in the 60 s by contemporary art. This paper proposes a new approach to "participative museology" with an eye on contemporary creations whose exhibitions loosely follow the approaches of the performing arts, and play on the "visitor-audienceproducer" relationship.

Key words: participatory museology, communication, living exhibitions 